\title{
Integrated computation model of lithium-ion battery subject to nail penetration
}

\author{
Binghe Liu ${ }^{\mathrm{a}, \mathrm{b}}$, Sha Yin ${ }^{\mathrm{a}, \mathrm{b}}$, Jun $\mathrm{Xu}^{\mathrm{a}, \mathrm{b}, \mathrm{c}, *}$ \\ ${ }^{a}$ Department of Automotive Engineering, School of Transportation Science and Engineering, Beihang University, Beijing 100191, China \\ ${ }^{\mathrm{b}}$ Advanced Vehicle Research Center (AVRC), Beihang University, Beijing 100191, China \\ ' State Key Laboratory of Nonlinear Mechanics (LNM), Institute of Mechanics, Chinese Academy of Sciences, Beijing 100190, China
}

\section{H I G H L I G H T S}

- A coupling model to predict battery penetration process is established.

- Penetration test is designed and validates the computational model.

- Governing factors of the penetration induced short-circuit is discussed.

- Critical safety battery design guidance is suggested.

\section{A R T I C L E I N F O}

\section{Article history:}

Received 29 May 2016

Received in revised form 16 August 2016

Accepted 17 August 2016

Available online 6 September 2016

\section{Keywords:}

Lithium-ion battery

Short circuit

Nail penetration

Coupled computation modeling
G R A P H I C A L A B S T R A C T

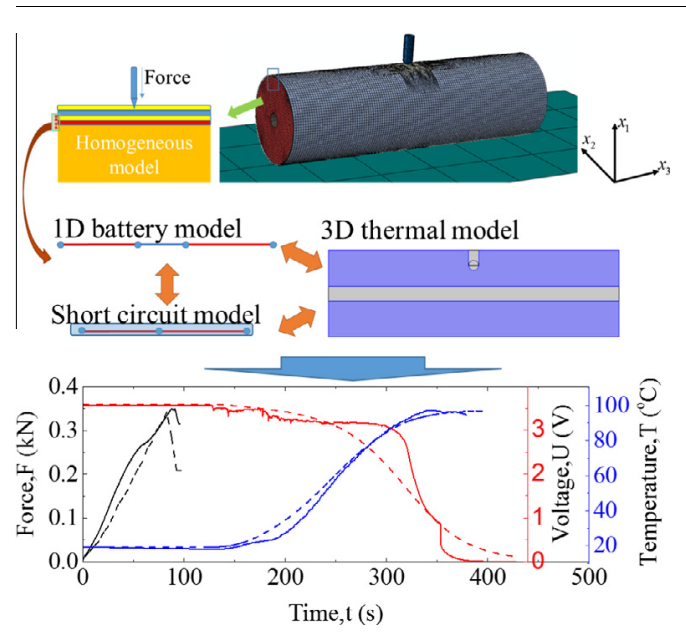

\begin{abstract}
A B S T R A C T
The nail penetration of lithium-ion batteries (LIBs) has become a standard battery safety evaluation method to mimic the potential penetration of a foreign object into LIB, which can lead to internal short circuit with catastrophic consequences, such as thermal runaway, fire, and explosion. To provide a safe, time-efficient, and cost-effective method for studying the nail penetration problem, an integrated computational method that considers the mechanical, electrochemical, and thermal behaviors of the jellyroll was developed using a coupled 3D mechanical model, a 1D battery model, and a short circuit model. The integrated model, along with the sub-models, was validated to agree reasonably well with experimental test data. In addition, a comprehensive quantitative analysis of governing factors, e.g., shapes, sizes, and displacements of nails, states of charge, and penetration speeds, was conducted. The proposed computational framework for LIB nail penetration was first introduced. This framework can provide an accurate prediction of the time history profile of battery voltage, temperature, and mechanical behavior. The factors that affected the behavior of the jellyroll under nail penetration were discussed systematically. Results provide a solid foundation for future in-depth studies on LIB nail penetration mechanisms and safety design.
\end{abstract}

(c) 2016 Elsevier Ltd. All rights reserved.

\footnotetext{
* Corresponding author at: Department of Automotive Engineering, School of Transportation Science and Engineering, Beihang University, Beijing 100191, China. E-mail address: junxu@buaa.edu.cn (J. Xu).
}

\section{Introduction}

The sales of electrical vehicles (EVs) have increased in the last few years [1], and lithium-ion batteries (LIBs) have been regarded 


\begin{tabular}{|c|c|c|c|}
\hline \multicolumn{4}{|c|}{ Nomenclature } \\
\hline C & heat capacity & $\varepsilon$ & strain \\
\hline$c$ & concentration & \multicolumn{2}{|c|}{$\varepsilon_{i}(i=1,2,3)$ principle strain } \\
\hline$D_{\text {eff }}$ & modified diffusion coefficient & $\varepsilon_{p}$ & plastic strain \\
\hline$d$ & displacement & & critical plastic strain \\
\hline$d_{f l}$ & separator failure displacement & $\bar{\varepsilon}_{f}$ & failure strain \\
\hline$d_{p n}$ & penetration displacement & $\dot{\varepsilon}$ & strain rate \\
\hline$d_{p n}(t)$ & penetration displacement with time & ${ }^{*} \dot{\varepsilon}$ & dimensionless plastic strain rate of winding nail \\
\hline E & Young's modulus & \multicolumn{2}{|c|}{$\varepsilon_{i k}$ and $\gamma_{i k}(i=1,2,3 ; k=1,2,3)$ strains in different directions } \\
\hline$E_{p}$ & in-plane modulus & $\kappa$ & electrical conductivity \\
\hline$E_{t}$ & transverse modulus & $\kappa_{\text {eff }}$ & modified electrical conductivity \\
\hline$E_{t g}$ & tangent modulus & $v$ & Poisson's ratio \\
\hline$E_{J}$ & joule heating energy & $v_{p t}$ & Poisson's ratio in in plane-transverse directions \\
\hline$E_{\text {eq }}$ & equilibrium potential & $v_{t p}$ & Poisson's ratio in transverse-in plane directions of the \\
\hline$F$ & Faraday's constant & & jellyroll \\
\hline$G_{p}$ & shear moduli in in-plane directions & $\rho$ & density \\
\hline$G_{t}$ & shear moduli in transverse directions & $\sigma$ & stress \\
\hline$H$ & height & $\sigma_{\text {Hill }}$ & Hill'48 equivalent stress \\
\hline$I$ & current & \multicolumn{2}{|c|}{$\sigma_{i k}$ and $\tau_{i k}(i=1,2,3 ; k=1,2,3)$ stresses in different directions } \\
\hline$I_{s t}$ & stable current & $\sigma_{\text {Mises }}$ & von Mises equivalent stress \\
\hline$\stackrel{i}{\rightarrow}$ & current density & & yield stress \\
\hline$j$ & current density & & potential \\
\hline$k$ & thermal conductivity & $1+\frac{\mathrm{d} \ln f}{\mathrm{~d} \ln c_{l}}$ & molar activity coefficient \\
\hline$k_{a}$ & thermal conductivity in angular direction & & \\
\hline$k_{r}$ & thermal conductivity in radial direction & \multicolumn{2}{|c|}{ Subscripts } \\
\hline$L$ & length & eff & modified \\
\hline$N$ & number & $e q$ & equilibrium \\
\hline$q_{a}$ & reaction heat & & \\
\hline$q_{j}$ & joule heat & \multicolumn{2}{|c|}{ Superscripts } \\
\hline$q_{r}$ & resistance heat & $a$ & anode \\
\hline$q_{i}$ & irreversible heat & $c$ & cathode \\
\hline$q$ & heat generation rate & & diffusion \\
\hline$R$ & radius & & jellyroll \\
\hline$R_{g}$ & gas constant & & electrolyte \\
\hline$R_{r}$ & resistance & $n c$ & negative collector \\
\hline$S$ & area & $n g$ & negative electrode \\
\hline$S O C$ & state of charge value & & penetration nail \\
\hline$T$ & temperature & & positive collector \\
\hline$t$ & time & $p c$ & positive electrode \\
\hline$t_{c}$ & current increasing time & ps & $\begin{array}{l}\text { positive electrode } \\
\text { electrode }\end{array}$ \\
\hline$t_{+}$ & transfer data & $s$ & $\begin{array}{l}\text { electrode } \\
\text { separator }\end{array}$ \\
\hline$V$ & voltage & se & $\begin{array}{l}\text { separator } \\
\text { short circuit part }\end{array}$ \\
\hline$v_{p n}$ & penetration speed & $s t$ & $\begin{array}{l}\text { short circuit part } \\
\text { winding nail }\end{array}$ \\
\hline$\delta$ & thickness & $w$ & \\
\hline$\epsilon$ & volume fraction & $3 D$ & 3D thermal model \\
\hline
\end{tabular}

as promising alternative energy sources for use in EVs [2,3]. LIB safety has become one of the main topics with regard to passenger safety because LIBs are frequently used in vehicles [4,5]. A thermal management system (TEM) is generally used to prevent overheating [6-8], but a short circuit may produce a significant amount heat that may cause thermal runaway [9].

Nail penetration, which mimics the penetration of foreign objects into LIB during use, has become a standard LIB safety evaluation method [10]. The immediate consequences of nail penetration include the occurrence of internal short circuit, which has potentially catastrophic consequences, such as fire and explosion [11-13]. In general, engineers must perform time-consuming and hazardous nail penetration tests $[10,14]$. These tests involve inserting a steel nail into LIB, thereby bridging the positive and negative electrodes within the jellyroll and causing local internal short circuit among the component interfaces of the nail and the jellyroll. Although nail penetration is essentially a mechanical loading process, it may involve electrochemical and thermal behaviors, which result from the strong entanglement of the multiphysical fields within the battery. Thus, a reasonable modeling framework is urgently required to understand and control the complicated mechanisms of a nail penetration-induced thermal runaway or fire.

Numerical modeling is an ideal substitution for real-world nail penetration testing. First, pioneering efforts have been made to understand the mechanical behavior of LIBs that are subjected to physical abuses, e.g., radial compression [15,16], indentation $[15,17]$, and bending [15] loads. The constitutive model for the jellyroll was first established by Greve and Fehrenbach [15] and Sahraei et al. [18] through homogeneous isotropic material treatment. Since then, Lai et al. [16] developed a representative volume element (RVE) model that considered each component of the jellyroll. Recently, an anisotropic model with coupled strain rate and state of charge (SOC) dependencies was proposed in Ref. [19]. Second, with regard to electrochemical behavior, a 1D battery model was 
first proposed by Newman et al. [20,21]. At present, such model is generally accepted as one of the most effective and accurate battery models. To improve the calibration of this battery model, the electrochemical properties of the cathode [11,22-24], anode [24-26], electrolyte [27,28], and separator [24,26] have been comprehensively studied. Third, internal short circuits have four types, as first discussed by Santhanagopalan et al. [29]. Fang et al. [11,30] conducted a numerical investigation of LIB short circuits using 3D model coupling that accounted for electrochemical and thermal influences. Recently, Chiu et al. [10] modeled thermal runaway behavior during the nail penetration process. Fourth, multiphysical simulations of LIBs have been developed by researchers [14,31-33]. These simulations typically involve electrochemicalthermal coupling $[11,31,32]$ or individual jellyroll components [34-36]. By contrast, Zhang et al. [37] were the first to suggest using a coupled mechanical-electrochemical-thermal method to study short circuit behavior under a mechanical load.

Nevertheless, a mechanical model with accurate material failure under mechanical abuse loadings has not yet been established, thereby preventing precise prediction of the nail and jellyroll interface contact. Furthermore, excessive heat that ultimately leads to thermal runaway produced by a high current density and the redox reaction of lithium is also highly dependent on the previous mechanical loading and battery model. Few studies have attempted to provide a reasonable coupled computation model to solve the aforementioned emerging engineering problem for nail penetration.

Accordingly, an integrated mechanical-electrochemical-thermal coupled modeling method is proposed in this study to investigate the nail penetration problem by integrating a 1D battery model, a 3D failure model, and a coupled short circuit model. Section 2 describes the development and validation of the three models using 18650 LIB as the modeling target of the experiments. Section 3 presents the typical nail penetration test conducted to validate the coupled computation model. Section 4 discusses the parametric studies performed to investigate the governing factors of LIB behavior during nail penetration. Section 5 provides a summary of the main results and conclusions of the study.

\section{Coupled computation modeling}

\subsection{Target 18650 lithium-ion battery}

Commercial 18650 LIBs (SONY), with a capacity of $2200 \mathrm{mAh}$, were used in this study as the experiment validation target battery. The anode and cathode materials are $\mathrm{Li}_{\mathrm{x}} \mathrm{C}_{6}$ (thickness: $\mathrm{mm}$ ) and $\mathrm{LiCoO}_{2}$ (thickness: $0.159 \mathrm{~mm}$ ), respectively. The charge voltage and cutoff voltage are $4.2 \mathrm{~V}$ and $2.5 \mathrm{~V}$, respectively. The basic dimensions are $\phi 18.6 \mathrm{~mm} \times 64.9 \mathrm{~mm}$. The jellyroll of the target LIB was studied individually. The dimensions of the jellyroll are summarized in Table 1.

\subsection{D mechanical model}

For simplicity, the 18650 LIB casing was not considered in both the experiments and the numerical modeling. In our previous study, a homogeneous anisotropic model of the jellyroll was developed [19] by considering SOC and dynamic effects; however, this homogenous model was incapable of indicating individual component failure. Thus, in the current study, the inner layers of the jellyroll were modeled using a homogeneous method to reflect the mechanical response of nail penetration, whereas the outer layers were configured layer by layer to describe the failure of each component (Fig. 1).
Table 1

Geometric parameters of the models.

\begin{tabular}{lll}
\hline Parameters & Values & Source \\
\hline $3 D$ geometry of the jellyroll & & \\
$R^{j}$ & $8.7 \mathrm{~mm}$ & Measured \\
$R^{w}$ & $2.5 \mathrm{~mm}$ & Measured \\
$\delta^{c}$ & $0.159 \mathrm{~mm}$ & Measured \\
$\delta^{a}$ & $0.165 \mathrm{~mm}$ & Measured \\
$\delta^{s e}$ & $0.018 \mathrm{~mm}$ & Measured \\
$\delta^{p c}$ & $0.013 \mathrm{~mm}$ & Measured \\
$\delta^{n r}$ & $0.019 \mathrm{~mm}$ & Measured \\
$H^{j}$ & $58.5 \mathrm{~mm}$ & Measured \\
$L^{j}$ & $700 \mathrm{~mm}$ & Measured \\
Geometry of the $1 D$ model & & \\
$L^{p s}$ & $\left(\delta^{c}-\delta^{p c}\right) / 2$ & Estimated \\
$L^{\text {ng }}$ & $\left(\delta^{a}-\delta^{n c}\right) / 2$ & Estimated \\
$L^{s e}$ & $\delta^{s e}$ & Estimated \\
$S^{j}$ & $L^{j} H^{j}$ & Estimated \\
\hline
\end{tabular}

\subsubsection{Modeling the inner layers of the jellyroll}

At present, the jellyroll is conventionally regarded as a macroscopic homogeneous cylinder with an initial small hole on the surface. In this study, the mechanical behavior difference between tension and compression is ignored. The main loading mode of nail penetration is compression, i.e., the mechanical composition within the $x_{1}-x_{2}$ plane is considered isotropic, and thus, the jellyroll is assumed to be transversely isotropic with respect to material behavior.

During the elastic deformation stage, the stress-strain relation can be expressed as follows [19]:

$\left\{\begin{array}{l}\varepsilon_{11}^{j} \\ \varepsilon_{22}^{j} \\ \varepsilon_{33}^{j} \\ \gamma_{12}^{j} \\ \gamma_{23}^{j} \\ \gamma_{31}^{j}\end{array}\right\}=\left[\begin{array}{cccccc}\frac{1}{E_{p}^{j}} & -\frac{v_{p t}^{j}}{E_{p}} & -\frac{v_{t p}^{j}}{E_{t}^{j}} & 0 & 0 & 0 \\ -\frac{v_{p t}^{j}}{E_{p}^{j}} & \frac{1}{E_{p}^{j}} & -\frac{v_{t p}^{j}}{E_{t}^{j}} & 0 & 0 & 0 \\ -\frac{v_{p t}^{j}}{E_{p}^{j}} & -\frac{v_{p t}^{j}}{E_{p}^{j}} & \frac{1}{E_{t}^{j}} & 0 & 0 & 0 \\ 0 & 0 & 0 & \frac{1}{G_{p}^{j}} & 0 & 0 \\ 0 & 0 & 0 & 0 & \frac{1}{G_{t}^{j}} & 0 \\ 0 & 0 & 0 & 0 & 0 & \frac{1}{G_{t}^{j}}\end{array}\right\}\left\{\begin{array}{c}\sigma_{11}^{j} \\ \sigma_{22}^{j} \\ \sigma_{33}^{j} \\ \tau_{12}^{j} \\ \tau_{23}^{j} \\ \tau_{31}^{j}\end{array}\right\}$

The Hill'48 failure criterion is used to define the mechanical yield surface of the jellyroll, which is written as

$\sigma_{\text {Hill }}^{j}=\sqrt{F^{j}\left(\sigma_{22}^{j}-\sigma_{33}^{j}\right)^{2}+G^{j}\left(\sigma_{33}^{j}-\sigma_{11}^{j}\right)^{2}+H^{j}\left(\sigma_{11}^{j}-\sigma_{22}^{j}\right)^{2}+2 L^{j} \tau_{23}^{j 2}+2 M^{j} \tau_{31}^{j 2}+2 N^{j} \tau_{12}^{j 2}}$,

where the six constants $F^{j}-N^{j}$ are the measures of anisotropy for each of the six components represented by the potentials $\left(R_{11}^{j}, R_{22}^{j}, R_{33}^{j}, R_{12}^{j}, R_{13}^{j}, R_{23}^{j}\right)$. The hardening law for the jellyroll was discussed in our previous study [19]. This law, coupled with the dynamic loading (strain rate) effect and the SOC effect, can be expressed as follows:

$\sigma^{j}=\left\{\begin{array}{ll}\left(B^{j} \varepsilon_{p}^{j n^{j}}+\sigma_{0}^{j}\right) \mathrm{e}^{c^{j} S O C}, & \varepsilon_{p}^{j} \leqslant \varepsilon_{p c}^{j} \\ \left.\sigma_{c}^{j}+B^{j}\left(\varepsilon_{p}^{j n^{j}}-\varepsilon_{p c}^{j n^{j}}\right) \mathrm{e}^{C^{j} S O C}\right] \mathrm{e}^{D^{j} \dot{\varepsilon}^{*}}, & \varepsilon_{p}^{j}>\varepsilon_{p c}^{j}\end{array}\right.$,

where $B^{j}, n^{j}, C^{j}$, and $D^{j}$ are the parameters determined through physical tests.

The equivalent strain failure shown below is chosen as the failure criterion:

$\bar{\varepsilon}^{j}=\bar{\varepsilon}_{f}^{j}$

where $\bar{\varepsilon}^{j}=\sqrt{\frac{2}{3}} \sqrt{\varepsilon_{1}^{j 2}+\varepsilon_{2}^{j 2}+\varepsilon_{3}^{j 2}}$. 

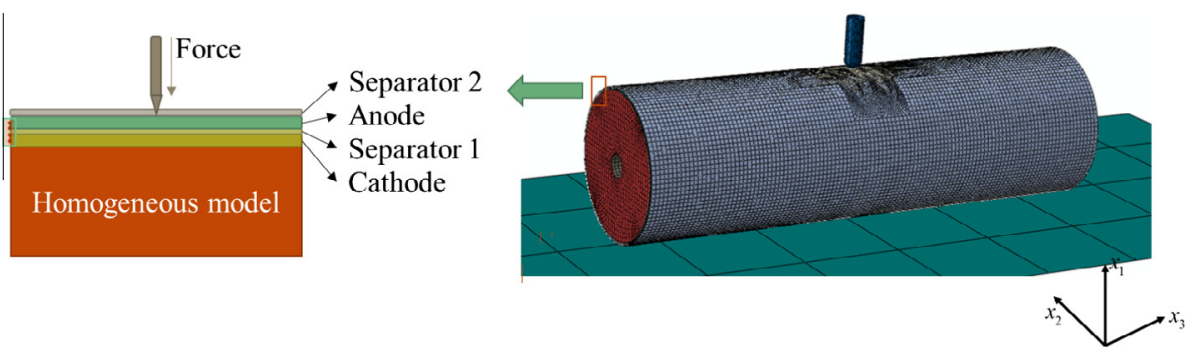

Fig. 1. Geometry of the 3D failure model.

In summary, the constitutive model is defined through 19 parameters, which are as follows:

- 6 elastic constants $\left(E_{p}^{j}, E_{t}^{j}, v_{p t}^{j}, v_{t p}^{j}, G_{p}^{j}, G_{t}^{j}\right)$;

- 6 parameters that define the yield surface $\left(R_{11}^{j}, R_{22}^{j}, R_{33}^{j}, R_{12}^{j}\right.$, $\left.R_{13}^{j}, R_{23}^{j}\right)$;

- 6 parameters that describe strain hardening $\left(\sigma_{0}^{j}, \varepsilon_{p c}^{j}, B^{j}, C^{j}, D^{j}, n^{j}\right)$; and

- 1 parameter that describes the failure criterion $\left(\bar{\varepsilon}_{f}^{j}\right)$.

Meanwhile, 18 parameters were previously determined in Ref. [19], and failure strain was established based on $\bar{\varepsilon}_{f}^{j}=0.55$ by comparing the experiment and simulation values. The parameter values are summarized in Table 2.

\subsubsection{Modeling the cathode}

The cathode is regarded as an isotropic model for nail penetration even though it is slightly anisotropic in the $x_{1}-x_{2}$ direction [17]. The elastic-power hardening model [38] is chosen for modeling based on the tension test results and expressed as follows:
$\left\{\begin{array}{ll}\sigma^{c}=E^{c} \varepsilon^{c} & \text { when } \sigma^{c} \leqslant \sigma_{0}^{c} \\ \sigma^{c}=A^{c} \varepsilon^{c n^{c}} & \text { when } \sigma^{c}>\sigma_{0}^{c}\end{array}\right.$,

where $A^{c}$ and $n^{c}$ are the material constants fitted from the experiment results in Ref. [17]. In addition, the von Mises criterion is used to define the yield surface as follows:

$\sigma_{\text {Mises }}^{c}=\sqrt{\left(\sigma_{22}^{c}-\sigma_{33}^{c}\right)^{2}+\left(\sigma_{33}^{c}-\sigma_{11}^{c}\right)^{2}+\left(\sigma_{11}^{c}-\sigma_{22}^{c}\right)^{2}+2 \tau_{23}^{c 2}+2 \tau_{31}^{c 2}+2 \tau_{12}^{c 2}}$.

The equivalent strain failure $\bar{\varepsilon}_{f}^{c}$ is determined from tension tests [17]. The values of the parameters are summarized in Table 2.

\subsubsection{Modeling the anode}

The anode is also regarded as an isotropic material, and the elastic-linear hardening model [38] is proposed and expressed as follows:

$\left\{\begin{array}{ll}\varepsilon^{a}=\frac{\sigma^{a}}{E^{a}} & \text { when } \sigma^{a} \leqslant \sigma_{0}^{a} \\ \varepsilon^{a}=\frac{\sigma_{0}^{a}}{E^{a}}+\frac{1}{E_{t g}^{a}}\left(\sigma^{a}-\sigma_{0}^{a}\right) & \text { when } \sigma^{a}>\sigma_{0}^{a}\end{array}\right.$,

Table 2

Summaries of material parameters.

\begin{tabular}{|c|c|c|c|c|c|}
\hline Parameters & Cathode & Separator & Anode & Jellyroll & $\begin{array}{l}\text { Winding nail } \\
{[41]}\end{array}$ \\
\hline Density & $4678 \mathrm{~kg} / \mathrm{m}^{3}[22,23]$ & $900 \mathrm{~kg} / \mathrm{m}^{3}[42]$ & $2270 \mathrm{~kg} / \mathrm{m}^{3}[24-26]$ & $2163.1 \mathrm{~kg} / \mathrm{m}^{3 \mathrm{a}}$ & $7850 \mathrm{~kg} / \mathrm{m}^{3}$ \\
\hline Elastic constants & $E^{c}=2940 \mathrm{MPa}[17]^{\mathrm{a}}$ & $E^{s e}=262.2 \mathrm{MPa}[17]^{\mathrm{a}}$ & $E^{a}=5372 \mathrm{MPa}[17]^{a}$ & $\begin{aligned} E_{t}^{j} & =1500 \mathrm{MPa} \\
G_{p}^{j} & =217 \mathrm{MPa} \\
G_{t}^{j} & =300 \mathrm{MPa} \\
E_{p}^{j} & =500 \mathrm{MPa}[19]\end{aligned}$ & $E^{w}=207 \mathrm{GPa}$ \\
\hline Poisson' radio & $v^{c}=0.3[17]$ & $v^{s e}=0.3[39]$ & $v^{a}=0.3[17]$ & $v_{p t}^{j}=0.15 v_{t p}^{j}=0.3[19]$ & $v^{w}=0.3$ \\
\hline Strain hardening & $\begin{array}{l}E_{t}^{c}=185 \mathrm{MPa} \\
\sigma_{0}^{c}=14.7 \mathrm{MPa}[17]^{\mathrm{a}}\end{array}$ & $\begin{array}{l}A^{s e}=108.3 \mathrm{MPa} n^{s e}=0.46 \\
\sigma_{0}^{s e}=47.9 \mathrm{MPa}[17]^{\mathrm{a}}\end{array}$ & $\begin{aligned} A^{a} & =32.84 \mathrm{MPa} \\
n^{a} & =0.2 \\
\sigma_{0}^{a} & =8.38 \mathrm{MPa}[17]^{\mathrm{a}}\end{aligned}$ & $\begin{array}{l}\sigma_{0}^{j}=0.8 \mathrm{MPa} C^{j}=1.103 \\
\varepsilon_{p c}^{j}=0.2 B^{j}=930 \mathrm{MPa} \\
D^{j}=0.02 n^{j}=3.4[19]\end{array}$ & $\begin{array}{l}\sigma_{0}^{w}=0.35 \mathrm{GPa} \\
B^{w}=0.37 \mathrm{GPa} \\
C^{w}=0.062 \\
n^{w}=0.348\end{array}$ \\
\hline Yield surface & & $\begin{array}{l}(1,0.162,0.162,0.28,1.73,1.73) \\
{[17]^{a}}\end{array}$ & & $\begin{array}{l}(12.25,1,1,12,12,1.7) \\
{[19]}\end{array}$ & \\
\hline Failure strain & $\bar{\varepsilon}_{f}^{c}=0.0066[17]^{a}$ & $\bar{\varepsilon}_{f}^{s e}=1[17]^{\mathrm{a}}$ & $\bar{\varepsilon}_{f}^{a}=0.05[17]^{a}$ & & \\
\hline Electrical conductivity & $\kappa^{a}=10 \mathrm{~S} / \mathrm{m}[11]$ & $\kappa^{s e}\left(c_{\mathrm{LiPF}_{6}}, T\right)[27,28]$ & $\kappa^{a}=100 \mathrm{~S} / \mathrm{m}[24-26]$ & & \\
\hline $\begin{array}{l}\text { Diffusion coefficient } \\
\text { Maximum concentration }\end{array}$ & $\begin{array}{l}D^{c}=5 \times 10^{-13}[22,23] \\
c_{e q}^{c}=56,250 \mathrm{~mol} / \mathrm{m}^{3} \\
{[22,23]}\end{array}$ & $D^{S e}\left(c_{\mathrm{LiPF}_{6}}, T\right)[27,28]$ & $\begin{array}{l}D^{a}(\mathrm{~T})[24-26] \\
c_{e q}^{a}=31,507 \mathrm{~mol} / \mathrm{m}^{3} \\
{[24-26]}\end{array}$ & & \\
\hline Parameters & Cathode & Separator & Anode & Jellyroll & Wind nail \\
\hline Heat capacity & $\begin{array}{l}C^{c}=1269.2 \mathrm{~J} /(\mathrm{kg} \mathrm{K}) \\
C^{p c}=875 \mathrm{~J} /(\mathrm{kg} \mathrm{K}) \\
{[24,26,42]}\end{array}$ & $\begin{array}{l}C^{s e}=1978.16 \mathrm{~J} /(\mathrm{kg} \mathrm{K}) \\
{[24,26,42]}\end{array}$ & $\begin{array}{l}C^{a}=1437.4 \mathrm{~J} /(\mathrm{kg} \mathrm{K}) \\
C^{n c}=385 \mathrm{~J} /(\mathrm{kg} \mathrm{K}) \\
{[24,26,42]}\end{array}$ & $C^{j}=1347.4 \mathrm{~J} /(\mathrm{kg} \mathrm{K})^{\mathrm{a}}$ & \\
\hline $\begin{array}{l}\text { Effective thermal expansion } \\
\text { coefficient }\end{array}$ & $\alpha^{c}=9.615 \times 10^{-6}[42]$ & $\alpha^{s e}=13.32 \times 10^{-5}[42]$ & $\alpha^{a}=4.06 \times 10^{-6}[42]$ & & \\
\hline Thermal conductivity & $\begin{array}{l}k^{c}=1.58 \mathrm{~W} /(\mathrm{m} \mathrm{K}) \\
k^{n c}=170 \mathrm{~W} /(\mathrm{m} \mathrm{K}) \\
{[24,26,42]}\end{array}$ & $k^{s e}=0.344 \mathrm{~W} / \mathrm{m} \mathrm{K}[24,26,42]$ & $\begin{array}{l}k^{a}=1.04 \mathrm{~W} /(\mathrm{m} \mathrm{K}) \\
k^{p c}=198 \mathrm{~W} /(\mathrm{m} \mathrm{K}) \\
{[24,26,42]}\end{array}$ & $\begin{aligned} k_{a}^{j} & =28.2 \mathrm{~W} /(\mathrm{m} \mathrm{K}) \\
k_{r}^{j} & =1.066 \mathrm{~W} /(\mathrm{m} \mathrm{K})^{\mathrm{a}}\end{aligned}$ & \\
\hline
\end{tabular}

\footnotetext{
${ }^{\text {a }}$ Estimated from the data.
} 
where $E^{a}$ and $E_{t g}^{a}$ are fitted from the experiment results in Ref. [17]. The yield surface is defined well using the von Mises criterion. Moreover, the equivalent strain failure $\bar{\varepsilon}_{f}^{a}$ determined by the tension experiment is chosen as the failure criterion. The parameter values are summarized in Table 2.

\subsubsection{Modeling the separator}

Celgard 2400, which is highly anisotropic, is used as the separator material $[39,40]$. Young's modulus $E^{\mathrm{se}}=216.2 \mathrm{MPa}$ is obtained from the stress-strain curve in Ref. [17], and Poisson's ratio is set to $v^{\text {se }}=0.3$ [39]. The Hill' 48 criterion is used for the yield surface as follows:
The main equations used are the mass balance and charge balance of the electrolyte $[45,46]$, as follows:

$\epsilon^{l} \frac{\partial c^{l}}{\partial t}=-D_{e f f}^{l} \nabla c^{l}+\frac{i^{l} t_{+}}{F}$

$i^{l}=-\kappa_{e f f}^{l} \nabla \varphi_{l}+\frac{2 \kappa_{e f f}^{l} R_{g} T}{F}\left(1+\frac{\mathrm{d} \ln f}{\mathrm{~d} \ln c^{l}}\right)\left(1-t_{+}\right) \nabla \ln c^{l}$.

Additional details regarding the modeling can be found in Ref. [20]. The produced reaction, joules, resistance, and irreversible heat of the battery are expressed as follows [45]:

$\sigma_{\text {Hill }}^{\text {se }}=\sqrt{F^{s e}\left(\sigma_{22}^{s e}-\sigma_{33}^{s e}\right)^{2}+G^{s e}\left(\sigma_{33}^{s e}-\sigma_{11}^{s e}\right)^{2}+H^{s e}\left(\sigma_{11}^{s e}-\sigma_{22}^{s e}\right)^{2}+2 L^{s e} \tau_{23}^{s e} 2+2 M^{s e} \tau_{31}^{s e}+2 N^{s e} \tau_{12}^{s e} 2}$,

where $F^{s e}-N^{s e}$ are confirmed by the potentials $\left(R_{11}^{s e}, R_{22}^{s e}, R_{33}^{s e}, R_{12}^{s e}, R_{13}^{s e}, R_{23}^{s e}\right)$, which are obtained from the experiment curves in Ref. [17]. The selected hardening model is also the power hardening model, which is shown as follows:

$\sigma^{s e}=A^{s e} \varepsilon^{s e} n^{s e}$ when $\sigma^{s e}>\sigma_{0}^{s e}$,

where $A^{s e}$ and $n^{\text {se }}$ are the material constants fitted from the experiment in Ref. [17].

The strain failure criteria are also used for the separator [37] with failure strain anisotropy [17]. The expression $\bar{\varepsilon}_{f}^{s e}=1$ is chosen to describe the onset of failure [17]. The mechanical parameters of the separator are summarized in Table 2.

\subsubsection{Modeling the winding nail}

The winding nail is made of steel, the hardening model of which is described using the Johnson-Cook model [41]:

$\sigma^{w}=\left(\sigma_{0}^{w}+B^{w} \varepsilon_{p}^{w n^{w}}\right)\left(1+C^{w} \ln ^{*} \dot{\varepsilon}^{w}\right)$

where ${ }^{*} \dot{\varepsilon}_{w}=\frac{\dot{\varepsilon}_{w}}{\varepsilon_{w 0}}$ is the dimensionless plastic strain rate with $\dot{\varepsilon}_{0}^{w}=1 \mathrm{~s}^{-1}$ as a reference strain rate; and $\sigma_{0}^{w}, B^{w}$ and $C^{w}$ are the parameters determined in Ref. [41]. The parameters are summarized in Table 2.

The finite element (FE) model for the jellyroll includes three parts: the winding nail, the homogeneous model for the jellyroll, and the outer layers. The winding nail and the outer layers are modeled using the shell element (C3D4 type in ABAQUS), and the homogeneous model uses the solid element (C3D8R type in ABAQUS). Element size is smaller within the vicinity of the penetration area. The model has a total of 188,649 elements (Fig. 1).

\section{3. $1 D$ battery model}

The classical 1D battery model established by Newman et al. $[20,21]$ was adopted and implemented in the numerical computation platform COMSOL Multiphysics [43]. Although the Newman model does not precisely describe the physics of battery electrochemical processes [44], it may accurately predict voltage change and heat generation under charge/discharge conditions, which are highly important for the coupled short circuit model. The 1D battery model can also represent the average electrochemical and thermal properties of the jellyroll. Thus, this model is chosen for this study to simplify computations. The 1D model has three parts: a negative electrode, a separator, and a positive electrode.
$q_{a}=\int_{0}^{L} i\left(\phi^{s}-\phi^{l}-E_{e q}\right) \mathrm{d} x$

$q_{j}=\int_{0}^{L} \kappa_{e f f}^{s}\left(\frac{\delta \phi^{s}}{\delta x}\right)^{2}+\kappa_{e f f}^{l}\left(\frac{\delta \phi^{l}}{\delta x}\right)^{2}+\kappa_{e f f}^{D}\left(\frac{\delta \ln c_{l}}{\delta x}\right)\left(\frac{\delta \phi_{l}}{\delta x}\right) \mathrm{d} x$

$q_{r}=I^{2} \frac{R_{r}}{S_{j}}$,

$q_{i} \approx 0$,

where $S^{j}=2 H^{j} L^{j}$, and $I$ is the current equal to $i S^{j}$. The total heat of the system is the sum of Eqs. (13)-(16).

The geometric parameters of the 1D model are summarized in Table 1 . In the $1 \mathrm{D}$ model, the positive and negative current collectors are insignificant because of small resistances. Similar to the modeling method presented in Section 2.1, the 1D battery model uses half of the thickness of the cathode and the anode, as well as the entire thickness of the separator. The cross section of this model is set to twice the unfolded area of the jellyroll.

\subsection{Short circuit modeling}

The short circuit model contains a 1D battery model, a representative short circuit model, and a 3D thermal model. The relationship among the three models including variable and parameter transferring logic, is illustrated in Fig. 2. The representative short circuit model is proposed to predict the excessive heat caused by short circuit. Assuming no explosion of the positive/negative collectors during nail penetration, the dominant cathode-anode short circuit mode is chosen of four internal short circuit modes [29].

For the selected internal short circuit model, the mechanical failure of the separator is the primary reason for the short circuit $[5,29,37,40,47]$. The short circuit resistances of the cathode, anode, and nail are configured in series. The nail has high electrical conductivity (nearly $10^{6} \mathrm{~S} / \mathrm{m}^{2}$ ), and the head produced by the nail is ignored in this analysis. The geometry of the $1 \mathrm{D}$ representative short circuit model is depicted in Fig. 2. The model only contains a cathode and an anode with lengths $L^{p s}$ and $L^{\text {ng }}$, respectively. The cross section $S^{\text {st }}$ is related to the radius of the puncture nail, which is

$S^{s t}=\pi R^{n l 2}$. 


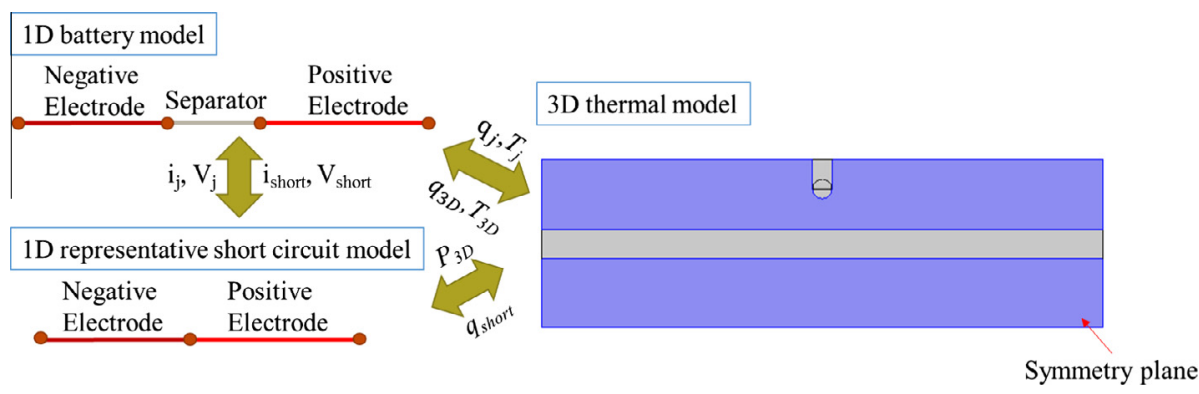

Fig. 2. Geometry of the 1D battery model and the short circuit model.

The governing equations for the electrical behavior are listed as follows [37]:

$\vec{j}=\kappa^{s t} \vec{\nabla} \phi$

$\vec{E}=-\vec{\nabla} \phi$

$E_{J}=\frac{\vec{j} \cdot \vec{j}}{\kappa^{s t}}$

The 3D thermal model is used to calculate heat conduction. A half model and the symmetry boundary conditions are used to simplify the computation. This model contains three components: the jellyroll, the short circuit area, and air. An open boundary is set for air, and a symmetric boundary is set for the symmetry plane (Fig. 2).

The governing equation for the thermal model is $[48,49]$

$\rho C \frac{\partial T}{\partial t}=\nabla \cdot(k \nabla T)+q$.

On the one hand, the 1D battery model and the representative short circuit model are coupled through the cell voltage $V^{j}$ and the current density $i^{j}$ of the battery model, and the voltage $V^{s t}$ and current density $i^{\text {st }}$ of the short circuit model. This quantitative relationship is expressed as

$V^{j}=V^{s t}$

$i^{j} A^{j}=N^{s t} i^{s t} S^{s t}$

where $N^{s t}$ is the number of short circuit parts in parallel (dependent on the time/displacement of the nail), which can be estimated using the following functions:

$N^{s t}(t)=1+\left[\frac{d_{p n}(t)-d_{f l}}{\left(1-\bar{\varepsilon}_{f}^{j}\right)\left(L^{n g}+L^{s e}+L^{p s}\right)}\right]$,

where [ ] is a round down function; $d_{p n}(t)$ is the penetration displacement with time; $d_{f l}$ is the separator failure displacement; $t$ is time; and $d_{p n}(t)$ is related to the penetration velocity, which is expressed as follows:

$d_{p n}(t)-d_{f l}=v_{p n} t$.

On the other hand, the 1D battery model and the 3D thermal models are coupled with the heat production rate $q^{j}$ and the temperature $T^{j}$ of the battery model, as well as the loading heat rate $q^{3 D}$ and the average temperature $T^{3 D}$ of the thermal model. This relationship is described as

$q^{3 D}=q^{j}$ and $T^{j}=T^{3 D}$.

Meanwhile, the short circuit representative model and the 3D thermal model are bridged with the heat production rate $q^{\text {st }}$ of the short circuit model and the overall heat rate $P^{3 D}$. This relationship is expressed as

$P^{3 D}=q^{s t} S^{s t}\left(L^{n g}+L^{p s}\right) N^{s t}(t) / 2$.

\section{Results}

\subsection{Model validation}

During the validation experiments, the jellyrolls were extracted from the LIBs as described in Section 2.1. An INSTRON 5966 universal material testing machine was used as the test platform. The loading speeds for both compression tests and nail penetration tests were set to $1 \mathrm{~mm} / \mathrm{min}$. The nail was ellipsoid-shaped with an end radius of $1 \mathrm{~mm}$. Charging and discharging conditions of $0.3 \mathrm{C}$ and $0.4 \mathrm{C}$, respectively, were implemented. During the experiment, the voltage and surface temperature (at four monitoring points) of the jellyroll were recorded using an Agilent 34410A digital voltmeter and temperature sensors, respectively.

\subsubsection{Validation of the $3 D$ mechanical model}

To ensure convergence within the modeling, three models were established with (i) only a homogenous jellyroll part; (ii) a onelayer set of cathode, anode, and separator, together with the rest of the homogenous jellyroll; and (iii) a two-layer set, together with the rest of the homogenous jellyroll. Fig. 3(a) shows the comparison between the compression experiment and the three modeling results. A reasonably good fit was observed, and material/structure failure was accurately predicted. The exact moments of the contact and material failure must be identified for nail penetration problems, and thus, the 3D model with a one-layer set with two layers of separators was adopted.

\subsubsection{Validation of the $1 D$ battery model}

Without losing generality, Fig. 3(b) shows the calibration of the 1D battery model with the charging/discharging tests of the cell. A high correlation was observed, thereby validating the 1D battery model against the target 18650 LIB.

\subsubsection{Validation of the short circuit model}

A nail penetration experiment was conducted to calibrate the established short circuit model. The total penetration displacement was $4 \mathrm{~mm}$. As shown in Fig. 3(c), reasonable agreements were achieved for both voltage and temperature time history profiles. In particular, the critical temperature rise and voltage drop points i.e., $t=125 \mathrm{~s}$, were precisely determined. The simulation data exhibit a small deviation with the experimental data when time is over $200 \mathrm{~s}$. The present model does not reflect the temperature dependence of the mechanical properties because of the lack of experiment data. 

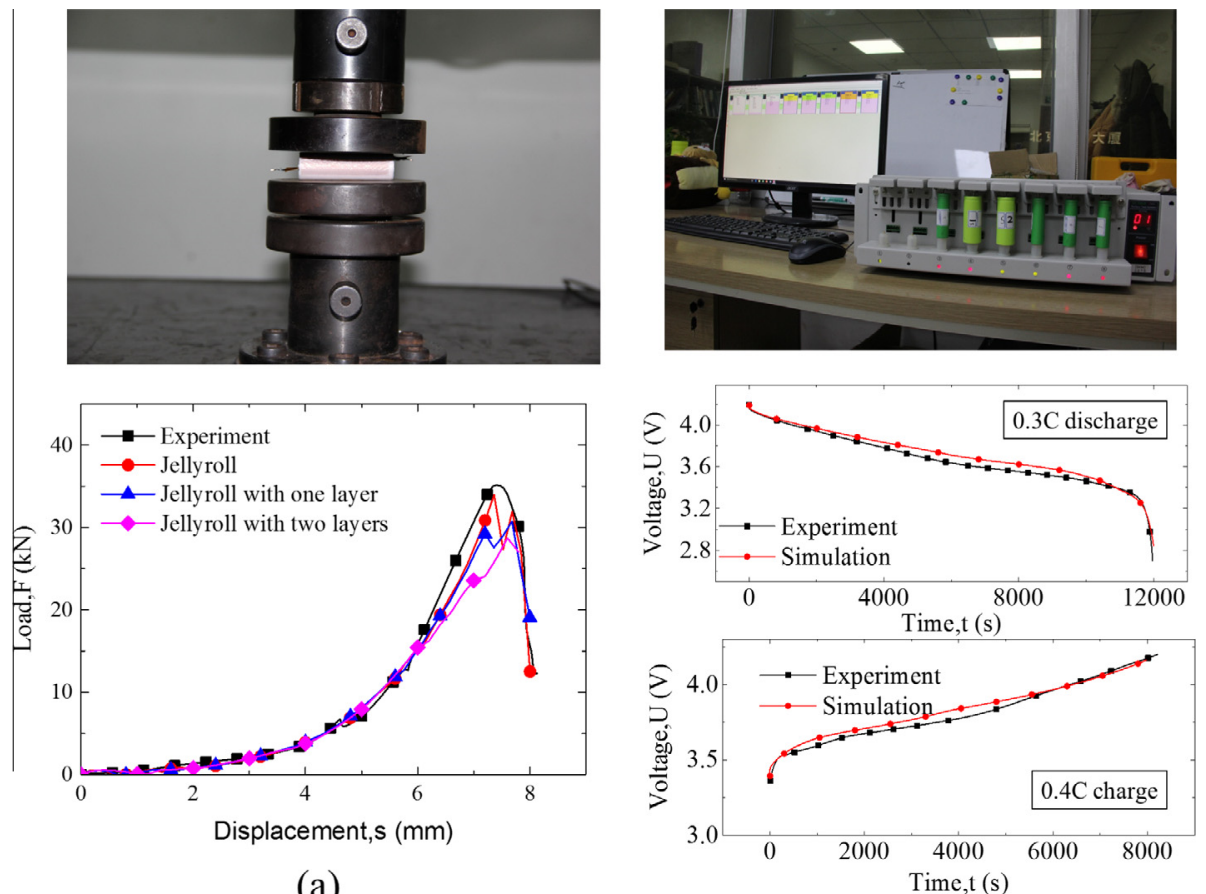

(a)

(b)
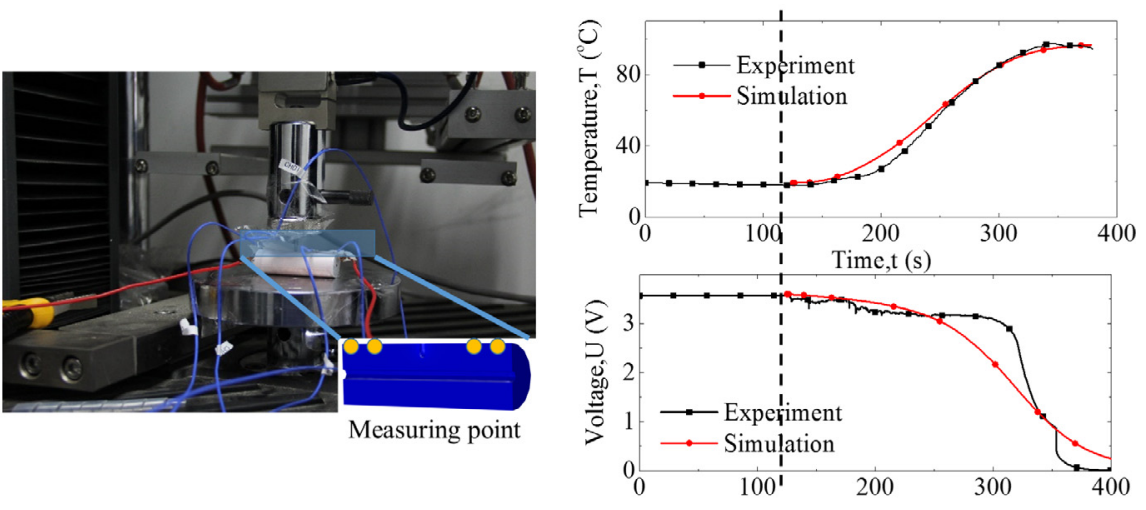

(c)

Time,t (s)

Fig. 3. (a) Load-displacement curve of the experiments and simulations using the 3D failure model in compression. (b) Voltage-time curves of the experiments and simulations using the 1D battery model in the charging/discharging tests. (c) Temperature and voltage-time curves of the experiments and simulations using the 1D battery model in the nail penetration test.

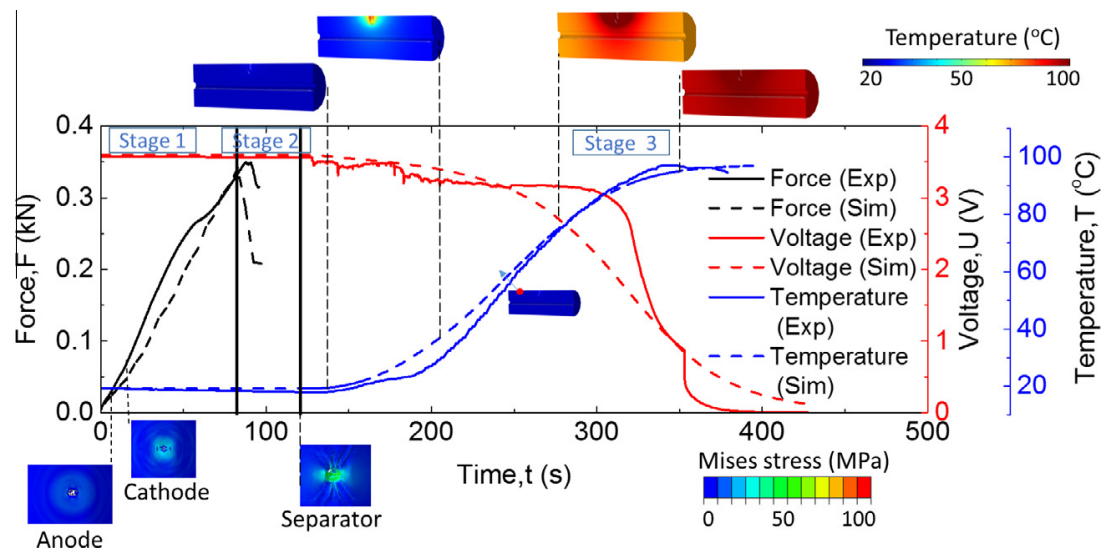

Fig. 4. Force, voltage, and maximum increased temperature-time curves of the jellyroll under nail penetration. The failure figure of the components and the temperature field distribution of the jellyroll are also depicted. 


\subsection{General results}

The overall mechanical, electrochemical, and thermal behaviors of the jellyroll can be divided into three stages based on the force, voltage, and temperature time history curves illustrated in Fig. 4. In Stage I (the mechanical deformation stage), the jellyroll experienced elastic and plastic mechanical deformations until it buckled while sustaining nearly constant electrochemical properties, except for a minor voltage increase reported in Ref. [50]. The cathode and the anode in the outer layers progressively failed. In Stage II (the penetration stage), after the buckling of the jellyroll structure (during which the anode and the cathode were also fractured), the separator with a large failure strain began to bear the load. As the displacement caused by the nail continued, the separator was penetrated once it reached mechanical failure strain. The cutoff point for this stage was the failure point of the outer layer of the separator. This critical point was predicted well by the 3D mechanical model compared with the tests. In Stage III (the short circuit stage), the cathode-anode short circuit began once the separator failed. Voltage decreased and temperature increased. The gradient of the voltage and temperature change increased as a function of time because the involved short circuit components, i.e., $N^{\text {st }}(t)$ of Eq. (25), increased with further penetration displacement. As shown in Fig. 4, the temperature of the short circuit area increased sharply at the beginning, thereby causing the overall temperature of the cell to increase through heat conduction. These findings agree well with those of the experiment. A voltage drop was also observed in Stage III, thereby demonstrating reasonable consistency with the voltage change profile during the experiment. The deviation with the experimental data can be attributed to the shortcoming of the short circuit model, which results from the absence of a temperature-dependent mechanical behavior.

\section{Discussion}

To further explore the governing factors in LIB short circuit behavior during nail penetration, a series of parametric studies, including nail shape, nail size, SOC value, penetration speed, and penetration distance, was conducted using the aforementioned validated computation model. The parameter baselines were set as a spherical penetration nail with $R^{n l}=1 \mathrm{~mm}, S O C=0.6, v_{p n}=$ $1 \mathrm{~mm} / \mathrm{min}$, and $d_{p n}=4 \mathrm{~mm}$. Notably, when one parameter was changed, the others remained the same.

\subsection{Penetration with various nail shapes}

Four typical end shapes of nails were selected, namely, ellipsoid (with a short shaft radius equal to half of the long axis), flat, cone (with a cone angle of $90^{\circ}$ ), and sphere, as shown in Fig. 5(a). The mechanical responses for different nail shapes evidently differed because large contact areas with the jellyroll resulted in considerable reaction forces under the same nail displacement. By contrast, the cone-shaped nail demonstrated an early force drop because of the sharp nail tip, thereby imposing an elevated stress concentration.

This model may also be capable of predicting failure of the outer layers. In general, the outer cathode and the anode fail at a smaller displacement compared with that of the separator because of their lower mechanical failure strain values. The separator typically fails at relatively large strains (deformation), and in some cases, even after the overall buckling of the jellyroll. This observation indicates that the drop in mechanical load cannot be used as the indicator for the cathode-anode short circuit of the jellyroll. Similar qualitative phenomena were also observed in the bending and indentation mechanical tests, thereby showing that the internal short circuit occurred after the force drop, whereas the internal short circuit occurred before the buckling in compression tests in previous investigations $[18,50]$.

The failure displacements of the cathode and the anode did not vary among nail shapes, whereas the failure displacement of the separator did. Among the tested nail shapes, the spherical nail appeared to be the safest because of its blunt head, which probably delayed the failure of the separator, as shown in Fig. 5(b).

Different mechanical behaviors resulted in various electrochemical and thermal behaviors following the short circuit. Fig. 5 (c) depicts the current-time curves after the short circuit of each nail shape. All shapes demonstrated an increased current because of the number of involved short circuit separator layers, and then became stable until penetration ceased. Fig. 5(c) shows that the current of the jellyroll penetrated by the sphere nail is the first to become stable and has the lowest stable current value. This result can be attributed to the spherical nail having a larger short

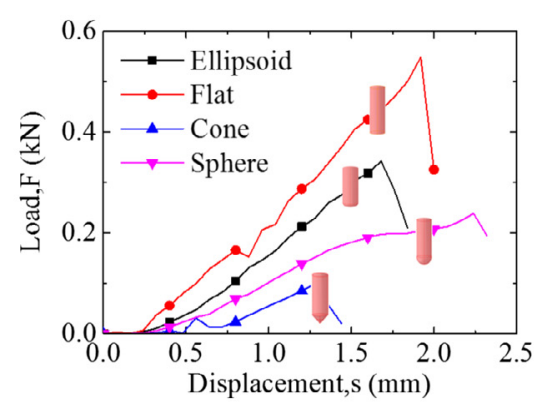

(a)

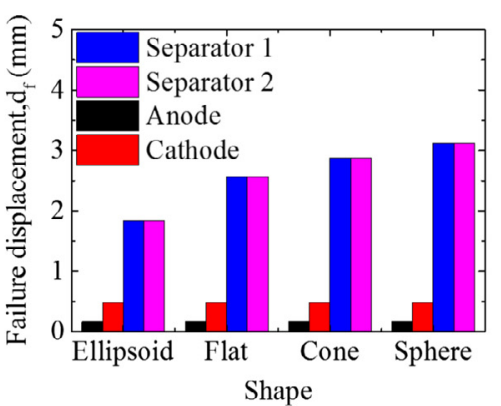

(b)

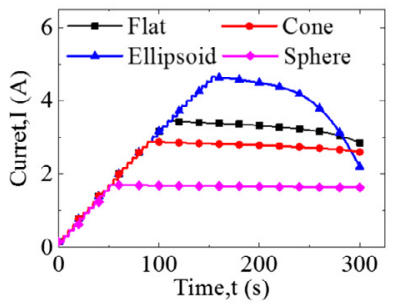

(c)

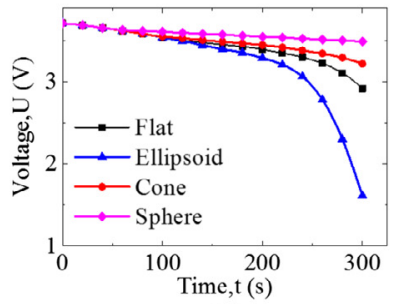

(d)

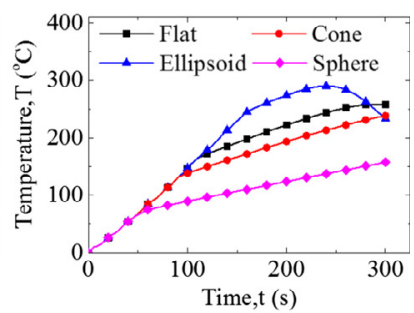

(e)

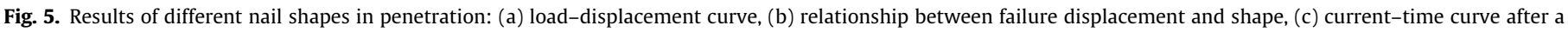
short circuit, (d) voltage-time curve after a short circuit, and (e) maximum increased temperature-time curve after a short circuit. 
circuit displacement. Thus, the failure of the separator value was the smallest at the same penetration displacement, which resulted in the lowest stable current. As shown in Fig. 5(c), the penetration of the ellipsoid nail has the shortest plateau-maintaining stable current because of the high current and the rapidly decreasing capacity of the jellyroll. Voltage dropped after short circuit occurred, and the voltage drop rate was highly dependent on the short circuit current. Thus, voltage dropped slowly at the beginning for a smaller short circuit and then increased gradually. An evident turning point of the voltage was observed as a result of the high potential change rate of the cathode and the anode at a low capacity $[22,25]$. Temperature evolution during nail penetration was determined based on current and voltage. For example, for the ellipsoid-shaped nail, the temperature decreased after the peak because of the rapid and steep drop in voltage, as shown in Fig. 5 (e). To summarize, the spherical nail caused the lowest short circuit current and the lowest temperature, while exhibiting improved capability to maintain voltage. Thus, spherical nail penetration is the least dangerous case for LIBs.

\subsection{Penetration with various nail sizes}

Evidently, small nails can easily penetrate the jellyroll, whereas large ones cause a large short circuit area.

Fig. 6(a) shows the load-displacement curves for the nail penetration simulation with nail sizes of $R^{n l}=0.25-1 \mathrm{~mm}$. Large nails clearly led to a considerable reaction force and large buckling displacement, in addition to internal short circuit displacement (i.e., separator failure displacement was also larger for larger nails). The relationship between internal short circuit displacement and nail size can be described as

$d_{f l}=3.3 R^{n l}$.

After an internal short circuit occurred, large nails caused large short circuit areas, thereby leading to large cross-section areas $S^{\text {st }}$. As shown in Fig. 6(c), the current increase rate is proportional to the square of the nail size, i.e., $R^{n l 2}$. The current increasing time was estimated using

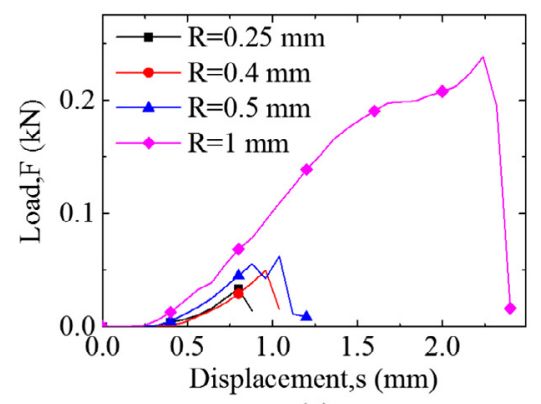

(a) $t_{c}=\frac{d_{p n}-3.3 R^{n l}}{v_{p n}}\left(d_{f l} \geqslant 3.3 R^{n l}\right)$.

The stable current $I_{\text {stable }}$ is proportional to $R^{n l 2} t_{c}$, which is expressed as

$I_{s t} \propto \frac{d_{p n}-3.3 R^{n l}}{v_{p n}} R^{n l 2}\left(d_{f l} \geqslant 3.3 R^{n l}\right)$.

The extreme value was reached when $R^{n l}=0.202 d_{p n}$, where $I_{s t}$ assumed a maximum value. Thus, the penetration displacement was a constant value. The parameter $I_{s t}$ initially increased, and then decreased as $R^{n l}$ increased, as illustrated in Fig. 6(c).

The voltage dropping rate and the temperature increasing rate increased prior to the achievement of a stable current with increasing $R^{n l}$. Once the current became stable, voltage drop and temperature gradient initially increased, and then decreased with increasing $R^{n l}$, as shown in Fig. 6(d) and (e).

\subsection{Penetration with various SOCs}

The mechanical behaviors of LIBs have been proven to be SOC dependent in previous studies [50], where LIBs with high SOCs have exhibited high stiffness. Consequently, the reaction force of LIBs with high SOCs is considerable during nail penetration, as shown in Fig. 7(a). Furthermore, force drop displacements also demonstrate a positive correlation with SOC values.

The failure displacement of the separator (short circuit displacement) presents a nearly linear relationship with SOC as follows:

$d_{f l}=3.12-0.7 S O C$.

A previous study [50] conducted compression experiments on LIBs with various SOCs, and the results showed that the internal short displacement and the SOC exhibited a linear relationship, with a highly similar slope to that in Eq. (31).

The current, voltage, and maximum increased temperaturetime curves are shown in Fig. 7(c)-(e). The current increasing rate slightly varied for different SOCs at varying initial voltages. The

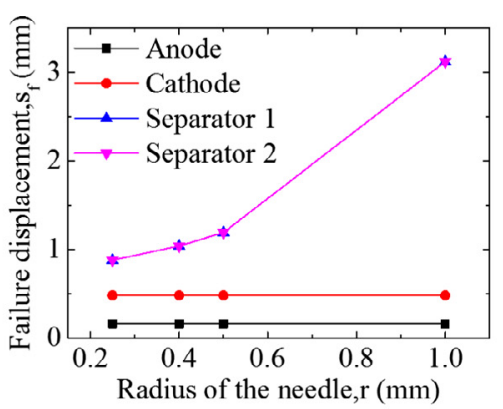

(b)

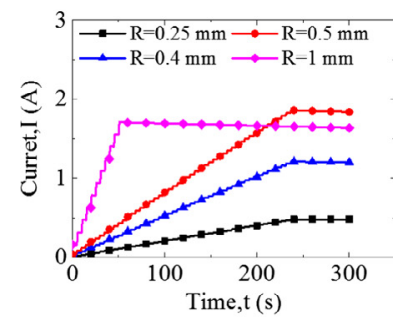

(c)

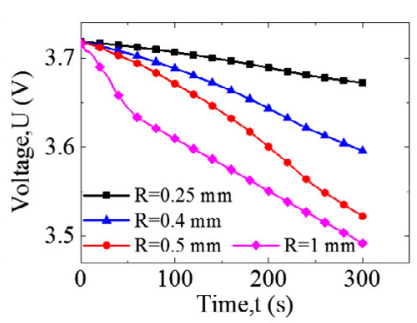

(d)

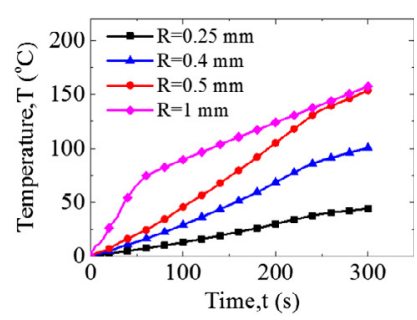

(e)

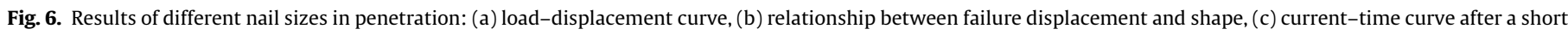
circuit, (d) voltage-time curve after a short circuit, and (e) maximum increased temperature-time curve after a short circuit. 


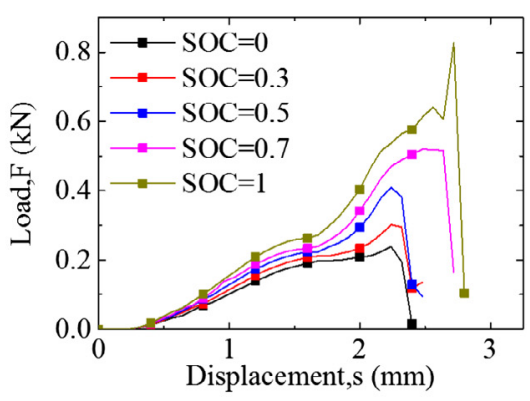

(a)

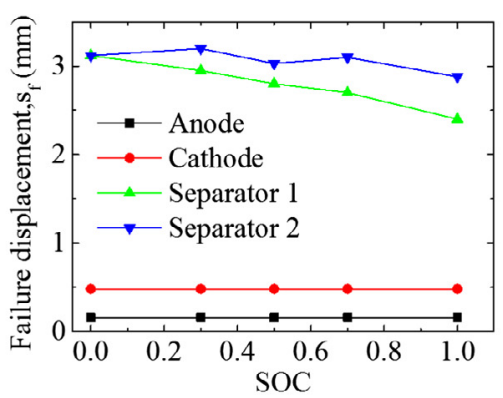

(b)

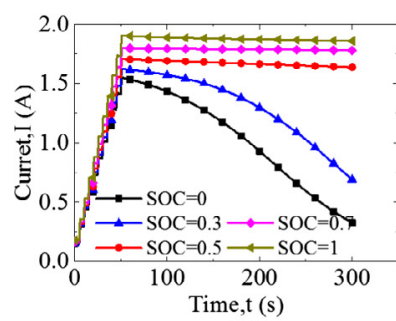

(c)

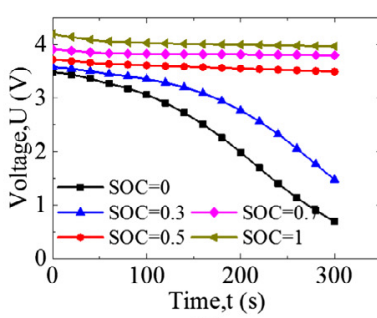

(d)

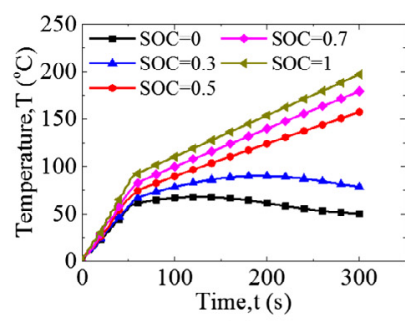

(e)

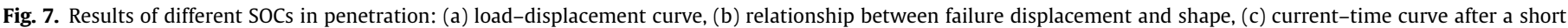
circuit, (d) voltage-time curve after a short circuit, and (e) maximum increased temperature-time curve after a short circuit.

capacity of the jellyroll in low SOCs (e.g., SOC $=0.2$ and 0.4 ) was depleted soon after the stable current was established, and voltage and current subsequently decreased as temperature changed from increasing to decreasing. However, for the jellyroll with a high SOC, a stable current was maintained for a sufficiently long period, such that the temperature exceeded the thermal runaway temperature, as shown in Fig. 7(e). The present model did not consider the thermal runaway behavior to include a discussion of temperatures exceeding $128^{\circ} \mathrm{C}[10]$.

\subsection{Penetration at various speeds}

Mechanically, penetration at high speeds caused the dynamic hardening of the jellyroll material. Compared with the quasi-static conditions, the reaction force at increased speeds was significantly higher (Eq. (10)). However, the short circuit displacement exhibited no evident regularity with penetration velocity, as shown in Fig. 8(a) and (b). Electrochemical and thermal behaviors were simulated at different loading speeds, including $0.01,0.1,1,10$, and $10,000 \mathrm{~mm} / \mathrm{s}$. The computation results are presented in Fig. 8(c)-(e). The stable current was the same for all four cases. However, the current increasing rate was highly related to penetration speed, although the current increasing rate demonstrated reduced sensitivity for speeds $>1 \mathrm{~mm} / \mathrm{s}$. Furthermore, the voltage and maximum temperature-time curves were nearly overlapping, thereby indicating that penetration speed did not significantly affect thermal and electrochemical behaviors once the loading speed was within a selected domain.

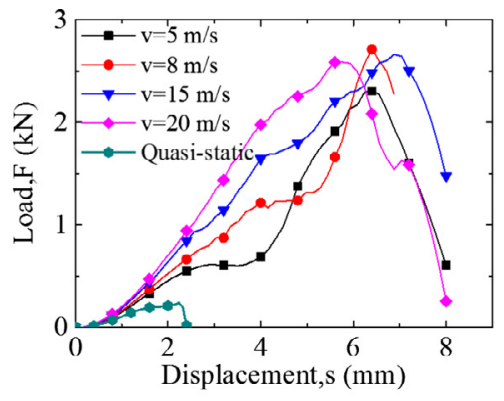

(a)

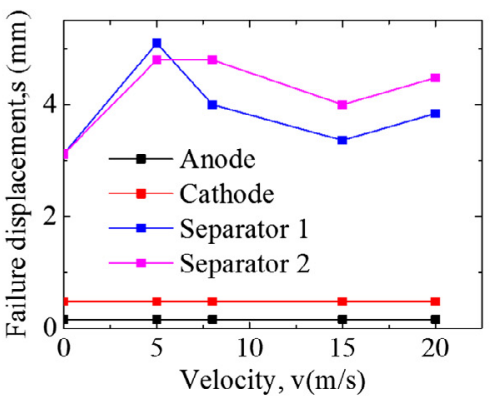

(b)

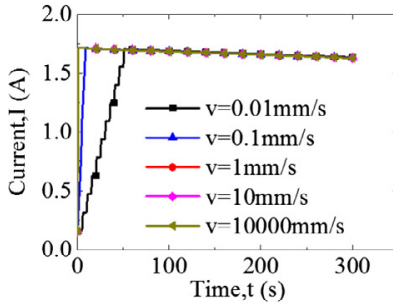

(c)

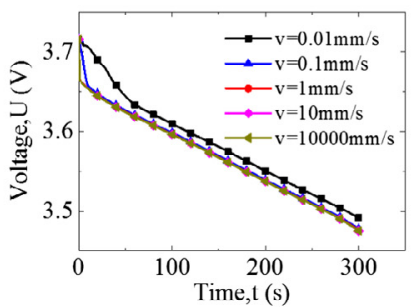

(d)

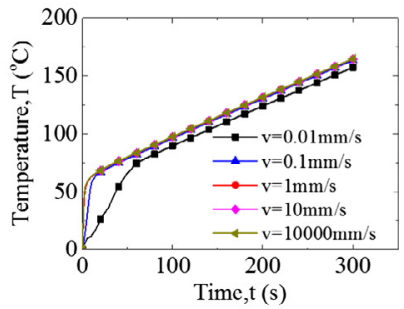

(e)

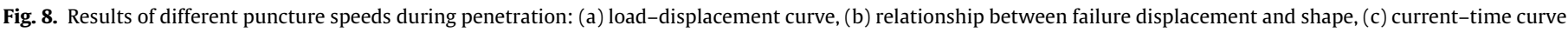
after a short circuit, (d) voltage-time curve after a short circuit, and (e) maximum increased temperature-time curve after a short circuit. 


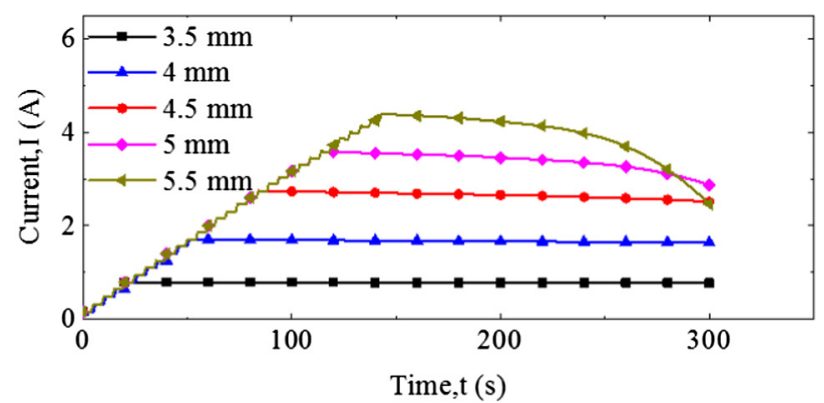

(a)

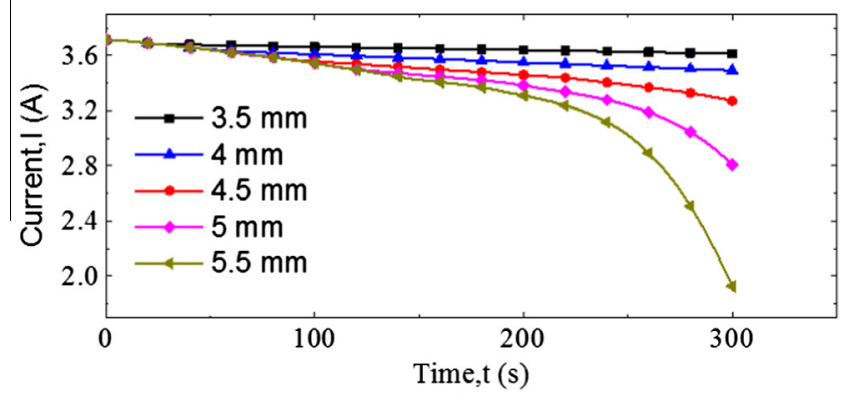

(b)

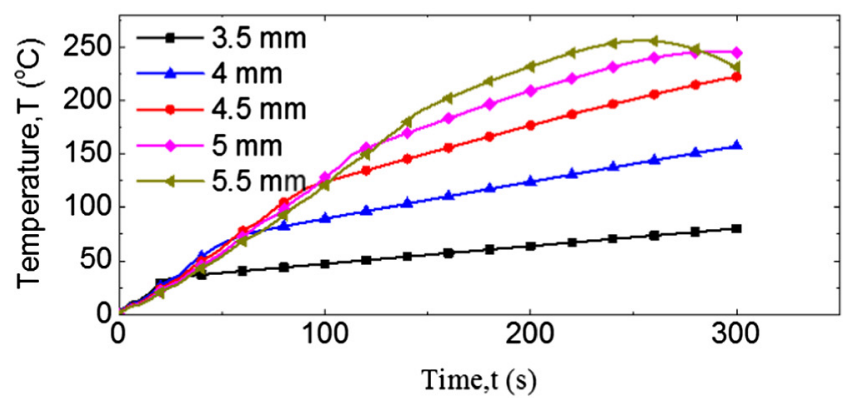

(c)

Fig. 9. Results of different penetration speeds: (a) current-time curve after a short circuit, (b) voltage-time curve after a short circuit, and (c) maximum increased temperature-time curve after a short circuit.

\subsection{Penetration at various nail displacements}

As shown by the established model, nail penetration displacement was a dominant factor of the stable current value because a large penetration displacement reflected numerous failed layers of separators. In this case, five discrete values of displacements (i.e., $d=3.5-5.5 \mathrm{~mm}$ ) were chosen for the study. Fig. 8(a) shows that the current increasing rates are the same before the stable current, and a large penetration displacement results in a high stable current value while maintaining a short plateau. Thus, voltage and maximum increased temperature were the same until the stable threshold value of the current was reached, as shown in Fig. 9 (b) and (c). A long puncture distance caused a sharp temperature increase and might have facilitated thermal runaway for the jellyroll.

\section{Concluding remarks}

Short circuit caused by nail penetration is an important issue in LIB safety. This study established an integrated mechanical-electro chemical-thermal behavior computation model by combining three models, namely, a 1D battery model, a 3D failure model, and a coupled short circuit model. The 1D battery model was modified based on the Newman model and used to calculate the electrochemical behavior of the jellyroll. The 3D failure model, which used a homogeneous model with external component layers, indicated internal short circuit displacement caused by the failure of the separator. Lastly, the coupled short circuit model was used to calculate electrochemical and thermal behaviors following an internal short circuit. These three models were validated separately, and the integrated model was also verified via experiments.

A series of parametric studies on various nail sizes, shapes, $\mathrm{SOC}$ values, penetration speeds, and nail tip displacements was conducted using the established integrated model. The following important results of the parametric studies were obtained for 18650 LIB.

- For different nail shapes, the order of the capability to deform the jellyroll is cone $>$ sphere $>$ ellipsoid $>$ flat. The order of short circuit displacement is sphere $>$ cone $>$ flat $>$ ellipsoid. The order of increasing temperature rate is sphere $<$ cone $<$ flat $<$ ellipsoid.

- For penetration nail sizes, short circuit displacement and needle radial exhibited the linear relationship of $d_{f l}=3.3 R^{n l}$. The extreme value of the stable current was achieved when $R^{n l}=0.202 d_{p n}$.

- For different SOCs, short circuit displacement presented a linear relationship with SOC, i.e., $d_{f l}=3.12-0.7 S O C$. For the jellyroll with a higher SOC, the current increasing rate and the stable current were slightly higher, and the stable current lasted longer.

- For different penetration distances, a large puncture distance caused a sharp temperature increase and might easily trigger the thermal runaway of the jellyroll.

- For different penetration speeds, short circuit displacement presented no difference. Penetration speed did not significantly affect thermal and electrochemical behaviors once loading speed was within the range of $1-10,000 \mathrm{~mm} / \mathrm{s}$.

These results can be used as bases for setting more reliable and appropriate penetration standards including test conditions, loadings, penetrator sizes and shapes, for various engineering applications. On the other hand, the coupled mechanicalelectrochemical-thermal simulation method can be used to guide the safety design of lithium-ion batteries for various service conditions.

\section{Acknowledgement}

This work is financially supported by the Fundamental Research Funds for the Central Universities, Beihang University; the startup fund for "Zhuoyue 100" titled professor, Beihang University; the Academic Excellence Foundation of BUAA for PhD Students and Opening fund of State Key Laboratory of Nonlinear Mechanics, Institute of Mechanics, Chinese Academy of Sciences.

\section{References}

[1] Zhou Y, Wang M, Hao H, Johnson L, Wang HW, Hao H. Plug-in electric vehicle market penetration and incentives: a global review. Mitig Adapt Strateg Glob Change 2015:20:777-95.

[2] Wang CY, Zhang G, Ge S, Xu T, Ji Y, Yang XG, et al. Lithium-ion battery structure that self-heats at low temperatures. Nature 2016;529. 515-+.

[3] Ping P, Wang Q Huang P, Sun J, Chen C. Thermal behaviour analysis of lithiumion battery at elevated temperature using deconvolution method. Appl Energy 2014;129:261-73.

[4] Feng X, Weng C, Ouyang M, Sun J. Online internal short circuit detection for a large format lithium ion battery. Appl Energy 2016;161:168-80.

[5] Chen J, Sun T, Qi Y, Li X. A coupled penetration-tension method for evaluating the reliability of battery separators. ECS Electrochem Lett 2014;3:A41-4. 
[6] Ling Z, Wang F, Fang X, Gao X, Zhang Z. A hybrid thermal management systen for lithium ion batteries combining phase change materials with forced-air cooling. Appl Energy 2015;148:403-9.

[7] Rao Z, Wang Q, Huang C. Investigation of the thermal performance of phase change material/mini-channel coupled battery thermal management system. Appl Energy 2016;164:659-69.

[8] Wang T, Tseng KJ, Zhao J, Wei Z. Thermal investigation of lithium-ion battery module with different cell arrangement structures and forced air-cooling strategies. Appl Energy 2014;134:229-38.

[9] Feng XN, He XM, Ouyang MG, Lu LG, Wu P, Kulp C, et al. Thermal runaway propagation model for designing a safer battery pack with $25 \mathrm{Ah}$ $\mathrm{LiNi}_{\mathrm{x}} \mathrm{Co}_{\mathrm{y}} \mathrm{Mn}_{\mathrm{z}} \mathrm{O}_{2}$ large format lithium ion battery. Appl Energy 2015;154:74-91.

[10] Chiu K-C, Lin C-H, Yeh S-F, Lin Y-H, Chen K-C. An electrochemical modeling of lithium-ion battery nail penetration. J Power Sources 2014;251:254-63.

[11] Fang W, Ramadass P, Zhang Z. Study of internal short in a Li-ion cell-Il. Numerical investigation using a 3D electrochemical-thermal model. J Power Sources 2014;248:1090-8.

[12] Hammami A, Raymond N, Armand M. Runaway risk of forming toxic compounds. Nature 2003:424:635-6.

[13] Feng X, He X, Ouyang M, Lu L, Wu P, Kulp C, et al. Thermal runaway propagation model for designing a safer battery pack with $25 \mathrm{Ah}$ $\mathrm{LiNi}_{\mathrm{x}} \mathrm{Co}_{\mathrm{y}} \mathrm{Mn}_{\mathrm{z}} \mathrm{O}_{2}$ large format lithium ion battery. Appl Energy 2015;154: 74-91.

[14] Zhao R, Liu J, Gu J. Simulation and experimental study on lithium ion battery short circuit. Appl Energy 2016;173:29-39.

[15] Greve L, Fehrenbach C. Mechanical testing and macro-mechanical finite element simulation of the deformation, fracture, and short circuit initiation of cylindrical lithium ion battery cells. J Power Sources 2012;214:377-85.

[16] Lai WJ, Ali MY, Pan J. Mechanical behavior of representative volume elements of lithium-ion battery modules under various loading conditions. J Power Sources 2014;248:789-808.

[17] Sahraei E, Kahn M, Meier J, Wierzbicki T. Modelling of cracks developed in lithium-ion cells under mechanical loading. RSC Adv 2015:5:80369-80.

[18] Sahraei E, Campbell J, Wierzbicki T. Modeling and short circuit detection of 18650 Li-ion cells under mechanical abuse conditions. J Power Sources 2012;220:360-72.

[19] Xu J, Liu B, Wang X, Hu D. Computational model of 18650 lithium-ion battery with coupled strain rate and SOC dependencies. Appl Energy 2016;172:180-9.

[20] Doyle CM. Design and simulation of lithium rechargeable batteries [Ph.D. thesis]. University of California; 1995.

[21] Fuller TF, Doyle M, Newman J. Simulation and optimization of the dual lithium ion insertion cell. J Electrochem Soc 1994;141:1-10.

[22] Tang M, Albertus P, Newman J. Two-dimensional modeling of lithium deposition during cell charging. J Electrochem Soc 2009;156:A390-9.

[23] Stephenson DE, Hartman EM, Harb JN, Wheeler DR. Modeling of particleparticle interactions in porous cathodes for lithium-ion batteries. J Electrochem Soc 2007;154:A1146-55.

[24] Gerver RE, Meyers JP. Three-dimensional modeling of electrochemical performance and heat generation of lithium-ion batteries in tabbed planar configurations. J Electrochem Soc 2011;158:A835-43.

[25] Srinivasan V, Newman J. Design and optimization of a natural graphite/iron phosphate lithium-ion cell. J Electrochem Soc 2004;151:A1530-8.

[26] Kumaresan K, Sikha G, White RE. Thermal model for a Li-ion cell. J Electrochem Soc 2008; 155:A164-71.

[27] Nyman A, Behm M, Lindbergh G. Electrochemical characterisation and modelling of the mass transport phenomena in LiPF6-EC-EMC electrolyte. Electrochim Acta 2008:53:6356-65.
[28] Zavalis TG, Behm M, Lindbergh G. Investigation of short-circuit scenarios in a lithium-ion battery cell. J Electrochem Soc 2012:159:A848-59.

[29] Santhanagopalan S, Ramadass P, Zhang J. Analysis of internal short-circuit in a lithium ion cell. J Power Sources 2009;194:550-7.

[30] Ramadass P, Fang W, Zhang Z. Study of internal short in a Li-ion cell I. Test method development using infra-red imaging technique. J Power Sources 2014;248:769-76.

[31] Guo M, Kim G-H, White RE. A three-dimensional multi-physics model for a Liion battery. J Power Sources 2013;240:80-94.

[32] Kim GH, Pesaran A, Smith K, Lee KJ, Santhanagopalan S. Multi-domain modeling of lithium-ion batteries encompassing multi-physics in varied length scales. J Electrochem Soc 2011;158:A955-69.

[33] Franco AA. Multiscale modelling and numerical simulation of rechargeable lithium ion batteries: concepts, methods and challenges. RSC Adv 2013;3:13027-58.

[34] Wu W, Xiao XR, Huang XS, Yan ST. A multiphysics model for the in situ stress analysis of the separator in a lithium-ion battery cell. Comput Mater Sci 2014;83:127-36.

[35] Malavé V, Berger JR, Zhu H, Kee RJ. A computational model of the mechanical behavior within reconstructed $\mathrm{Li}_{\mathrm{x}} \mathrm{CoO}_{2}$ Li-ion battery cathode particles. Electrochim Acta 2014;130:707-17.

[36] Barai P, Mukherjee PP. Stochastic analysis of diffusion induced damage in lithium-ion battery electrodes. J Electrochem Soc 2013;160:A955-67.

[37] Zhang C, Santhanagopalan S, Sprague MA, Pesaran AA. Coupled mechanicalelectrical-thermal modeling for short-circuit prediction in a lithium-ion cell under mechanical abuse. J Power Sources 2015;290:102-13.

[38] Bertram A. Elasticity and plasticity of large deformations, vol. 6. Springer, Berlin; 2005. p. 205-25.

[39] Xu J, Wang L, Guan J, Yin S. Coupled effect of strain rate and solvent on dynamic mechanical behaviors of separators in lithium ion batteries. Mater Des 2016;95:319-28.

[40] Chen J, Yan Y, Sun T, Qi Y, Li X. Deformation and fracture behaviors of microporous polymer separators for lithium ion batteries. RSC Adv 2014:4:14904-14.

[41] Xu J, Liu B, Wang L, Shang S. Dynamic mechanical integrity of cylindrical lithium-ion battery cell upon crushing. Eng Fail Anal 2015;53:97-110.

[42] Shi DH, Xiao XR, Huang XS, Kia H. Modeling stresses in the separator of a pouch lithium-ion cell. J Power Sources 2011;196:8129-39.

[43] Multiphysics C. COMSOL multiphysics user guide (Version 4.3 a). COMSOL, AB; 2012

[44] Lai W, Ciucci F. Mathematical modeling of porous battery electrodes-revisit of Newman's model. Electrochim Acta 2011;56:4369-77.

[45] Newman J, Thomas-Alyea KE. Electrochemical systems. John Wiley \& Sons; 2012.

[46] Miranda D, Costa CM, Almeida AM, Lanceros-Mendez S. Computer simulations of the influence of geometry in the performance of conventional and unconventional lithium-ion batteries. Appl Energy 2016;165:318-28.

[47] Chen J, Yan Y, Sun T, Qi Y, Li X. Probing the roles of polymeric separators in lithium-ion battery capacity fade at elevated temperatures. J Electrochem Soc 2014;161:A1241-6.

[48] Holman JP. Heat transfer. McGraw-Hill; 1963.

[49] Merlin K, Delaunay D, Soto J, Traonvouez L. Heat transfer enhancement in latent heat thermal storage systems: comparative study of different solutions and thermal contact investigation between the exchanger and the PCM. Appl Energy 2016;166:107-16.

[50] Xu J, Liu B, Hu D. State of charge dependent mechanical integrity behavior of 18650 lithium-ion batteries. Sci Rep 2016;6:21829. 